\title{
ESTÁNDAR ORAL Y ENSEÑANZA DE LA PRONUNCIACIÓN DEL ESPAÑOL COMO PRIMERA LENGUA Y COMO LENGUA EXTRANJERA
}

\author{
Carme Carbó, Joaquim Llisterri, María Jesús Machuca, \\ Carme de la Mota, Montserrat Riera y Antonio Ríos \\ Universitat Autònoma de Barcelona \\ \{carbo|joaquim|maria|carme|montse|mestre\}@liceu.uab.es
}

\begin{abstract}
Resumen
The aim of this work is to analyse the teaching situation of Oral Standard Spanish. First, the indications on pronunciation present in the official and unofficial normative documents, as well as in the descriptive publications are revised. Secondly, it is presented the utility of applying this knowledge to the teaching of phonetics and to the teaching and correction of pronunciation as a part of the oral expression. In third place, the contexts in which it is necessary a good command of pronunciation are presented, and the available materials to achieve this goal are also examined.

The main conclusions are: the lack of official normative documents that collect the present uses of Spanish, the imprecision and incoherency of the curriculum objectives, the lack of exhaustive and systematic descriptions in the reference books for native speakers which, in many cases, even contain mistakes; the lack of proper material to learn Spanish pronunciation adopting a communicative approach.
\end{abstract}

La pronunciación es el gobierno de la voz y del cuerpo a partir de la dignidad de las cosas y de las palabras.

(Cicerón, De inventione, 1, 7, 9)

\section{Problemas para establecer un estándar oral del español}

¿Olimpiada u olimpiada? Cada cuatro años, a fuerza de escuchar el término en los medios de comunicación, recordamos que el español presenta en ocasiones variedades de pronunciación que se consideran igualmente correctas. Aunque la Real Academia Española manifieste su preferencia por la forma olimpiada en el Diccionario académico, ambas posibilidades prosódicas están reconocidas y las preferencias en el uso parecen ser consecuencia de la variación geográfica. No sucede así con pares del tipo intervalo-intérvalo o libidolibido, puesto que las formas esdrújulas son tildadas de "erróneas" y se sanciona su uso en situaciones comunicativas formales. Ante ejemplos como estos, el usuario puede sentirse desconcertado.

El interés por la pronunciación se remonta a los estudios clásicos de retórica y oratoria, especialmente a las recomendaciones presentes sobre actio y pronuntiatio en la obra de Cicerón y en las Institutio oratoria de Quintiliano. La ortoepía, definida en el Diccionario de la Real Academia (Real Academia Española, 2001) como el "arte de pronunciar correc- 
tamente" y la ortofonía, "la corrección de los defectos de la voz y de la pronunciación" forman parte de la ortología, el "arte de pronunciar correctamente y, en sentido más general, de hablar con propiedad".

Cada situación de uso condiciona en los hablantes la selección de unas formas de expresión verbal. En ámbitos formales, existe la necesidad social de construir un discurso oral adecuado que no provoque el rechazo o el descrédito. A pesar de tener un buen criterio de adecuación, el hablante puede descubrir que su competencia comunicativa es deficiente porque no domina el "arte" de la expresión oral. Necesitaría conocer cuáles son las propuestas de las instituciones a las que se reconoce capacidad prescriptiva para poder escoger en cada situación la pronunciación que crea más conveniente.

La noción de norma lingüística está inevitablemente asociada a conceptos como el prestigio, el alcance demográfico, la generalización y fijación en el uso, el ideal prototípico, el concepto de modelo o la voluntad prescriptiva. Para poder codificar la norma estándar de una lengua se requiere plantear, discutir y resolver los problemas derivados de la variación. La empleada en la enseñanza del español debería ser una norma lingüistica estándar panhispánica que representara un marco de referencia para los hablantes, que les permitiera identificarse como grupo lingüístico, que garantizara "una total disponibilidad para cualquier oyente en cualquier situación comunicativa" (Hernández Alonso, 2001) y que presentara una "corrección democráticamente conseguida y aceptada" (Alvar, 1983: 55).

Establecer el estándar de una lengua no es una tarea exenta de dificultades, y de gran complejidad en el caso del español. Al determinar los objetivos que rigen la elaboración del Diccionario panhispánico de dudas, la Real Academia afirma: "El español, por su carácter de lengua supranacional, constituye en realidad un conjunto de normas diversas que, no obstante, comparten una amplia base común". No obstante, la vasta extensión del territorio y el elevado número de hablantes, con características locales diferenciadas, exigen el reconocimiento de un estándar para cada una de las distintas zonas lingüísticas, peninsulares y extrapeninsulares. En lo que respecta a la pronunciación, no es fácil aceptar una única norma por la multitud de "acentos" del español.

En la codificación de cada fenómeno debe valorarse la posibilidad de una interferencia linguística, deben tenerse en cuenta el origen histórico y la expansión o regresión en el uso, la extensión geográfica, la valoración social y el empleo en función del registro o del grado de formalidad. Evidentemente, el estándar oral no debe ser un mero reflejo del escrito, ya que posee formas y usos distintos, y no ha de ser tampoco un calco de una de las múltiples situaciones de la comunicación oral: la coloquial. Debe formularse basándose en estudios de campo que tengan en cuenta la especificidad de la lengua oral y no tomando como única referencia la escritura. Para ello es imprescindible contar con descripciones cuidadosas de la situación lingüística en todo el ámbito.

La formulación de preceptos suele despertar reticencias en la comunidad de hablantes, puesto que en la determinación de lo que es correcto puede descubrirse el rechazo e incluso el desprecio de lo que no lo es. Así pues, las recomendaciones sobre la pronunciación deberían realizarse introduciendo matices, especificando si el ámbito de uso ha de ser general o si ha de ser exclusivo de una zona geográfica en la que existen normas específicas de uso. Parece prudente, por tanto, formular la norma de una manera flexible, que tenga en cuenta la adecuación comunicativa y que permita también la variedad de uso en función del grado de formalidad. 
Este trabajo se analiza la situación del estándar oral en la enseñanza del español. En el apartado 2 se revisan las indicaciones que se recogen sobre la pronunciación en los documentos normativos oficiales y no oficiales y en los descriptivos. En el apartado 3 se plantea la utilidad de aplicar estos conocimientos a la enseñanza de la fonética, y a la enseñanza y corrección de la pronunciación como parte integrante de la expresión oral. Finalmente, en los apartados 4 y 5 se presentan los contextos en los que se precisa el aprendizaje y el dominio de la pronunciación, y se examinan los materiales disponibles para ello.

\section{La pronunciación del español en los documentos normativos y no normativos}

\subsection{Documentos normativos oficiales}

La Real Academia Española - como reza en sus estatutos- es la institución encargada de velar por la unidad de la lengua en los cambios que experimente al adaptarse a las necesidades de los hablantes. Sus propuestas, una vez consensuadas por las Academias de la Lengua asociadas a la Española, constituyen una norma socialmente aceptada y se les atribuye carácter prescriptivo. Entre los documentos normativos se incluyen las orientaciones específicas, de ámbito restringido, que llevan a cabo algunas Academias.

La última gramática normativa publicada por la Real Academia data de 1931. Después de un largo período de tiempo, apareció como texto provisional, sin carácter vinculante, El Esbozo de una Nueva Gramática de la Lengua Española (Real Academia Española, 1973). Es una gramática descriptiva del español, de una variante neutra; no hay muchas referencias diatópicas y diastráticas, especialmente sobre las variedades propias de los países americanos, debido a una "información insuficiente en cuanto a su extensión y aceptación" (Real Academia Española, 1973: 5). Esta obra introduce cambios sustanciales en las divisiones tradicionales de materias. El apartado de Fonología reúne las antiguas Prosodia y Ortografía, ampliando notablemente sus contenidos. Constituye una descripción del sistema fonológico del español: fonemas, sonidos, sílaba, procesos fonológicos, fonotaxis, acento y entonación. Las referencias explícitas a la pronunciación -por ejemplo, las peculiaridades del acento y de las agrupaciones de sonidos en contacto- han de buscarse en los epígrafes dedicados a los temas de fonología correspondientes. Como en toda la obra, la variante lingüística descrita no está marcada por rasgos dialectales, y se mencionan fenómenos generalizados - por ejemplo, el yeísmo-; como modelo de entonación se toma el habla de la burguesía madrileña, culta y universitaria (Real Academia Española, 1973: §1.7).

Otras obras académicas, la Ortografia (Real Academia Española, 1999) y el Diccionario (Real Academia Española, 2001), contemplan fenómenos relacionados con la pronunciación, pero siempre vinculados a su repercusión en la norma ortográfica. En la Ortografia (Real Academia Española, 1999: §4.2), por ejemplo, se discute la realización como diptongo o hiato de los grupos vocálicos $<\mathrm{ui}>\mathrm{y}<\mathrm{iu}\rangle$, que incide en la colocación de la tilde. El Diccionario académico no incluye la pronunciación de los vocablos en la información lexicográfica -algo habitual en la tradición de la lengua española por la interpretación fónica regular de su ortografía-, solo hay referencias a casos excepcionales, por ejemplo, en los artículos correspondientes se describen la aspiración dialectal y en algunos extranjerismos de $h$, la doble interpretación fónica de $w$ y la pronunciación de $x$ como fricativa velar en 
los mexicanismos. El Diccionario académico no resuelve el problema de la pronunciación de los extranjerismos no adaptados ortográficamente (affaire, airbag, geisha, pizza...); de hecho, en la última edición no se han mantenido las notas que recogían la anterior (Real Academia Española, 1992) -por ejemplo, la aspiración de $h$ en hachís-y las propuestas de enmiendas, que eran consultables a través de internet -asi, por ejemplo, se discutía la vacilación que suscita la pronunciación de la palabra geisha, proponiéndose [géisa], dato finalmente no incluido-.

\subsection{Documentos normativos no oficiales y descriptivos}

Prescindiendo de las obras especializadas en la descripción fonética, entre los materiales descriptivos no normativos que son útiles para la enseñanza y el aprendizaje de la lengua oral se incluyen los tratados de ortología, los manuales de español correcto, los manuales de expresión oral, los manuales de estilo y los diccionarios de dudas.

Los manuales de pronunciación y los tratados de ortología no se pueden considerar estrictamente obras normativas, en tanto que no están escritas por la Academia. Las dos obras más conocidas y de mayor prestigio de la lengua española son el Manual de pronunciación española (1918) y el Compendio de Ortología española (1928) de Navarro Tomás. En ellos figuran valiosas observaciones pero no todas son pertinentes para el hablante actual ni reflejan las tendencias de todo el dominio hispánico.

El objetivo de los manuales de español correcto (por ejemplo, Gómez Torrego, 2002) es dar a conocer la norma académica teniendo en cuenta las desviaciones que los hablantes acostumbran a cometer; por tanto, aunque aparecen sistematizados los errores de pronunciación, no aparecen descripciones articulatorias de los sonidos, ni de los rasgos prosódicos: acento, entonación, ritmo, pausas, etc. Se da por supuesto que el lector es hablante nativo de la lengua y, obviamente, ya sabe cómo se realizan esos sonidos y cuáles son sus características prosódicas.

En los manuales de expresión oral (entre otros, Sánchez, 1983; García Madrazo y Moragón Gordon, 1989; Cuervo y Diéguez, 1991; Alcoba et alii, 2000; Gimeno et alii, 2000) se tratan todos los aspectos relacionados con los distintos niveles de análisis de la lengua, incluyendo la fonética y la fonología, además de las técnicas de expresión propias de la narración, la argumentación, el relato, la descripción, la entrevista, etc. En este tipo de materiales, en el apartado de fonética y fonología, puede encontrarse una breve descripción articulatoria de los sonidos, así como de los rasgos prosódicos. También se incluyen descripciones de la norma y de los errores más frecuentes que producen los hablantes.

El objetivo de los manuales de estilo de los medios de comunicación oral (Telemadrid, 1993; Mendieta, 1993; Radio y Televisión Andaluza, 1991; entre otros) es velar por la calidad de la lengua que utilizan sus profesionales. Generalmente, se dan recomendaciones para evitar los "vicios" prosódicos más frecuentes: dejo, tonillo, monotonía, afectación, etc. En algunas de estas obras, se describe brevemente la pronunciación de algunos sonidos y se presentan los errores más frecuentes, siguiendo, generalmente, la norma descrita por la Academia.

Los diccionarios de dudas (por ejemplo, Seco, 1998; Martínez de Sousa, 1996) resuelven algunas de las cuestiones gramaticales que resultan conflictivas en el uso. En cuanto a la pronunciación, tratan especialmente la de los términos extranjeros y la de los vulgarismos. 
En este tipo de obras no se hace ninguna referencia a los rasgos prosódicos, exceptuando los problemas de doble acentuación.

\section{Enseñanza de la expresión oral, enseñanza de la pronunciación, corrección fonética y enseñanza de la fonética}

Tras el análisis de la definición del estándar oral en español y de los principales documentos que ofrecen indicaciones sobre la norma de pronunciación, cabe plantearse el modo de llevar a cabo una acción formativa orientada a los potenciales usuarios de este estándar. Para ello, puede ser útil distinguir los siguientes ámbitos: enseñanza de la expresión oral, enseñanza y corrección de la pronunciación y enseñanza de la fonética.

La expresión oral forma parte de las destrezas o habilidades comunicativas clásicas que, junto con la comprensión oral, y la expresión y la comprensión escritas constituyen uno de los pilares de los diseños curriculares para la enseñanza de la lengua, tanto materna como extranjera. La adecuada realización de los elementos segmentales (la pronunciación de los sonidos) y de los suprasegmentales (el acento, la entonación, la velocidad de elocución, las pausas y el ritmo) debe considerarse prácticamente imprescindible para una buena expresión oral, pero no es sino uno de los elementos que configuran esta destreza. Otros componentes esenciales de la expresión oral son, por ejemplo, la adaptación a la situación comunicativa, la adecuación del registro y la distinción entre los diversos géneros orales.

La enseñanza de la pronunciación es, en cambio, una actividad orientada a conseguir una producción apropiada de los elementos segmentales y suprasegmentales de la lengua. En este sentido, se integra en la expresión oral, pero constituye un ámbito especializado que requiere, por parte del profesor, un buen conocimiento de la descripción fonética de la lengua. Durante el proceso de aprendizaje el alumno cometerá, inevitablemente, errores, y es en este momento cuando entra en juego la corrección fonética, un conjunto de estrategias que, partiendo del error del estudiante, consigan acercar sus realizaciones fonéticas a las deseadas.

Finalmente, aunque a veces se equiparen erróneamente fonética y pronunciación, debe señalarse que la enseñanza de la fonética es una reflexión metalingüística sobre el funcionamiento del sistema sonoro de la lengua. Se trata de un ámbito que tiene su lugar en los planes de estudio encaminados a la formación de especialistas en el análisis y en la descripción de la lengua, y que también tiene que formar parte del bagaje de quienes se dedican a la enseñanza de la pronunciación.

Así pues, la enseñanza de la pronunciación y la corrección fonética son dos actividades que se enmarcan en la enseñanza de la expresión oral, mientras que la enseñanza de la fonética proporciona al profesor las bases necesarias para llevarlas a cabo.

Estas reflexiones parecen válidas tanto en lo que se refiere al español como primera lengua como en lo que concierne al español como lengua extranjera. Es obvio que el contexto, el punto de partida y los objetivos en la enseñanza de la pronunciación difieren parcialmente en uno y otro caso, pero existen una serie de necesidades comunes como una definición precisa del estándar que ha de constituir el punto de llegada del aprendizaje y el conocimiento por parte del profesor de la descripción fonética de la lengua, de las técnicas para la enseñanza de la pronunciación y de las estrategias más adecuadas para la corrección de los errores que surgirán a lo largo del aprendizaje. 


\section{La enseñanza de la pronunciación en español como primera lengua}

\subsection{Contextos en los que se enseña la pronunciación en español: situación actual}

A pesar de la primacía que tiene la lengua oral sobre la escrita, en España, hasta la Reforma Educativa de la Ley orgánica de Ordenación General del Sistema Educativo de 1991 (LOGSE), las asignaturas de lengua se centraban básicamente en el estudio del código escrito y en la descripción formal de la lengua. Con la Reforma se propone un enfoque más comunicativo y la expresión oral adquiere más importancia. Algunos especialistas en la materia incluso insisten "en la necesidad de eliminar el predominio casi absoluto de las prácticas escritas en la enseñanza de la lengua y de atender programada y sistemáticamente al desarrollo de la oralidad" (Trigo Cutiño, 1998: 35).

En los primeros años de escolarización, en los que se enseña a leer, se muestran las relaciones entre las grafías y su pronunciación. Sin embargo, dado que en español, a diferencia de otras lenguas, como el inglés, estas relaciones tienden a la univocidad, si el estudiante no presenta retrasos en el aprendizaje de la lectura, apenas si se presta atención a la pronunciación, excepto si sirve como pretexto para tratar la ortografía.

La situación no es demasiado distinta en la enseñanza secundaria (tanto obligatoria como postobligatoria). El objetivo durante esta etapa es que el alumno adquiera un buen dominio de la expresión oral y los problemas de pronunciación apenas se tratan si esta no dificulta la comprensión o provoca problemas ortográficos. Así, según Vilà et alii (2002: 112 ), cuando los estudiantes acaban la enseñanza secundaria, en general, tienen la sensación de que -aparte de la lectura en voz alta- han realizado pocas actividades relacionadas con la expresión oral. Además, a menudo se tiende a evaluar la producción oral a partir de criterios propios de la lengua escrita, centrándose especialmente en los aspectos sintácticos. Asimismo, durante las actividades de lectura, muchas veces los profesores no saben cómo orientar a sus alumnos para que la mejoren. Obsérvese, a título de ejemplo, la falta de precisión en el planteamiento de las dificultades fónicas de la lengua oral que De Luca (1983: 33) presenta en su libro Didáctica de la lengua oral. Metodología de enseñanza y evaluación: "Se detectan en esta oportunidad todas las deficiencias fónicas del discurso: las articulaciones relajadas, las pronunciaciones exageradas que deforman sonidos, las entonaciones inadecuadas, etc."

En el caso de España, en la Exposición de motivos de la Ley Orgánica 10/2002, de 23 de diciembre, de Calidad de la Educación (publicada en el BOE el 24 de diciembre de 2002), se reconoce que los alumnos de Educación Secundaria "presentan graves deficiencias de expresión oral y escrita que están relacionadas con la falta de hábito de lectura". La ley establece que en la Educación Preescolar se debe atender "a las primeras manifestaciones de la comunicación y del lenguaje" (Cap. II, art. 10,5). En el nivel de Educación Infantil el objetivo es "desarrollar sus habilidades comunicativas orales e iniciarse en el aprendizaje de la lectura y de la escritura" (Cap. III, art. 12,2, e). Dejando a un lado los niveles de carácter voluntario, se dispone que durante la Educación Primaria se desarrollará la capacidad de "conocer y usar adecuadamente la lengua castellana y, en su caso, la lengua cooficial de la Comunidad Autónoma, en sus manifestaciones oral y escrita, así como adquirir hábitos de lectura" (Cap. IV, art. 15, 2, e). En etapa de Educación Secundaria Obligatoria se contribuirá a desarrollar en los alumnos la capacidad de "comprender y expresar con corrección, 
oralmente y por escrito, en la lengua castellana y, en su caso, en la lengua cooficial de la Comunidad Autónoma, textos y mensajes complejos, e iniciarse en el conocimiento, la lectura y el estudio de la literatura" (Cap. V, sección $1^{\text {a }}$, art. 22, 2, e).

Los esfuerzos dedicados a los ejercicios de pronunciación son mucho más evidentes en los primeros años de escolarización. A medida que el alumno avanza en edad y estudios la atención que se presta a la enseñanza de los aspectos fónicos de la lengua decrece. En realidad, si el discente no presenta retrasos en la adquisición del sistema fonológico, la pronunciación se trata básicamente como una herramienta al servicio de la ortografía; y la enseñanza de la pronunciación correcta u ortología apenas tiene presencia ni en la enseñanza secundaria ni en la superior. En la universidad, a pesar de la importancia que para el futuro profesional tendrá el uso efectivo de la lengua, no siempre se programan asignaturas dedicadas a mejorar las competencias comunicativas y, todavía resulta más infrecuente que se programe la enseñanza de la pronunciación y sus usos sociales. Aunque existen asignaturas en las que se trata la expresión oral y la elocución en estudios de enseñanza superior (especialmente en carreras relacionadas con la lengua), se da prioridad a la expresión escrita.

Por más que existe una conciencia de la necesidad de la expresión oral, y de que su enseñanza sea obligatoria en los distintos niveles preuniversitarios, una vez terminados los estudios reglados todavía se detectan deficiencias importantes. Los alumnos presentan dificultades para variar de registro. De modo análogo, les resulta difícil introducir matices mediante cambios de entonación y tienden a la monotonía melódica, especialmente en la lectura.

\subsection{Necesidades en campos profesionales relevantes}

El dominio de la expresión oral y, en concreto, de las variantes fónicas asociadas a los distintos usos de la propia lengua se requiere tanto en las profesiones que tienen la lengua como instrumento comunicativo (políticos, publicistas, abogados, etc.) como en las profesiones en las que la lengua se convierte en objeto de conocimiento (lingüistas, psicólogos, logopedas, etc.). Obviamente, en el segundo de los casos es igualmente necesario, si no más, mostrar un buen dominio de la expresión lingüística.

Al diseñar el currículo de los estudios que conducen al dominio de la lengua para su uso profesional dentro del marco del español para fines específicos, el objetivo es dotar al alumno de la competencia lingüística que se precisa para utilizarla como lengua de trabajo. Este objetivo, que tan evidente es cuando se trata de una segunda o una tercera lengua, debe ser también prioritario en la didáctica de la lengua propia, ya que acabará convirtiéndose también en instrumento de trabajo para la mayoría de los estudiantes. Para conseguir una comunicación oral efectiva es preciso tener una competencia lingüística adecuada a las necesidades derivadas de los distintos ámbitos en los que se produce la comunicación. En el Marco de referencia europeo para el aprendizaje, la enseñanza y la evaluación de lenguas (cap. 4: 47) se distingue entre el ámbito personal, el ámbito público, el ámbito profesional y el ámbito educativo, aunque no es raro que un ámbito se solape con otros.

Cuando el ejercicio de la profesión requiere hablar en público se hace preciso el conocimiento de la expresión oral formal y de las variantes fónicas que van a resultar adecuadas al entorno comunicativo. El hablante, en tanto que usuario consciente de la lengua, debe disponer de un amplio repertorio de registros fónicos y debe saber en qué situación resulta 
adecuado el empleo de cada uno. Por ejemplo, al expresarse oralmente en situaciones formales es imprescindible saber si el seseo es adecuado en todas las zonas de habla española o si su uso resulta pertinente solo en algunas de ellas.

La lengua oral formal se emplea como instrumento en las profesiones vinculadas a las ciencias humanas, por ejemplo, en la gestión cultural de administraciones públicas y privadas, en la mediación intercultural o en la dinamización cultural de los ayuntamientos y de otras entidades cívicas. Se precisa también en salones y ferias, en el mundo del turismo, de la hostelería y en cualquier entorno en el que se requieran las relaciones públicas. La lengua oral se emplea en el mundo de los negocios, de la economía, del comercio, de las finanzas, de los seguros. El dominio de la argumentación, de la persuasión y de las estrategias orales resulta esencial para políticos, diplomáticos, abogados. Los científicos de todos los campos del saber intercambian información con sus colegas y exponen los resultados de sus investigaciones en congresos en los que disponen de un tiempo muy limitado para realizar sus presentaciones.

Aunque los objetivos no sean necesariamente coincidentes, los lingüistas, los psicólogos del lenguaje, los logopedas, los maestros, los pedagogos y los profesionales de la interpretación convierten el conocimiento del sistema fónico de la lengua y de sus variedades segmentales y suprasegmentales en objeto de análisis. El conocimiento fonético y fonológico es esencial para despertar, desarrollar, evaluar o corregir la competencia comunicativa de otros hablantes. Además de comprender el funcionamiento del sistema de la lengua, deben conocer los mecanismos físicos asociados a la pronunciación y deben ser capaces de comparar las producciones de los sujetos estudiados con diversos patrones posibles de producción. Deben, por lo tanto, conocer las variantes sincrónicas de la lengua, los fenómenos derivados del contacto entre lenguas y las formas que resultan apropiadas en cada situación comunicativa.

Los locutores, los profesionales de los medios orales de comunicación, convierten la lengua en un medio y en un fin. Para los profesionales del teatro, del doblaje y del canto, el domino de la voz y de la dicción se convierte simplemente en arte.

Aunque no sucede así en todos los casos, la formación en cada uno de los ámbitos en los que el ejercicio de la profesión requiere hablar en público debería contemplar la didáctica de las habilidades comunicativas orales $y$, por consiguiente, la enseñanza de la pronunciación. Esta formación supondría continuar con los objetivos y métodos iniciados en la infancia. Deberían discutirse las variantes de uso existentes y los contextos en los que es conveniente emplearlas, y deberían proporcionarse pautas para poder expresarse con corrección en entornos formales (Kostouli, 2002: 11).

A pesar de la poca importancia que se da al aprendizaje de la pronunciación correcta dentro de la enseñanza reglada, parece adecuado considerar que todos los profesionales que utilicen la lengua como herramienta de trabajo deberían pronunciar correctamente y conocer las implicaciones lingüísticas, pragmáticas, culturales y sociales que la variación de los elementos segmentales y suprasegmentales conlleva.

En aquellas sociedades en las que conviven varias lenguas o existe una fuerte inmigración, se debe tener en cuenta que algunos de los problemas que se observan en el uso apropiado de los elementos fónicos pueden atribuirse a la inevitable interferencia lingüística. A este propósito, en el Marco de referencia europeo para el aprendizaje, la enseñanza y la evaluación de lenguas se afirma con contundencia que "todo el mundo sabe que la lengua 
de la enseñanza, incluso en Europa, no suele ser la lengua nativa de los alumnos". En la práctica diaria, el profesor de español como lengua extranjera y el profesor de español como lengua de enseñanza no nativa deben tener en cuenta las relaciones entre el sistema fónico del español y el de la lengua materna del alumno (Poch, 1999). De hecho, la convivencia entre lenguas o la enseñanza en una lengua no nativa son factores que dificultan todavía más la identificación de los usos sociales prestigiosos de ámbito general, los usos aceptables en ámbitos formales pero restringidos y los usos carentes de prestigio social.

Son muchos los casos de interferencia que pueden detectarse en el proceso de aprendizaje de la pronunciación:

a) La inexistencia de un determinado sonido del español en la lengua materna del alumno. El sonido palatal lateral, por ejemplo, no existe en inglés, por lo que es previsible que los estudiantes de español de habla inglesa tengan serias dificultades para producirlo.

b) La existencia de sonidos con una realización articulatoria cercana, pero no coincidente. Mientras que la realización del fonema / $t /$ en inglés es alveolar, la del español es dental.

c) La existencia de un mismo sonido en las dos lenguas pero con un uso social distinto. Así por ejemplo, el sistema fonológico vocálico español consta de cinco vocales mientras que el sistema del árabe formal solo tiene tres $(/ \mathrm{a} /, / \mathrm{i} /, / \mathrm{u} /)$. A pesar de que en el árabe coloquial de algunas zonas aparecen dichas vocales, los estudiantes árabes de español suelen tener problemas en la pronunciación de las vocales medias (/e/, $/ 0 /)$.

d) La existencia de un mismo sonido en las dos lenguas pero con distinta distribución. Aunque en español estándar, por ejemplo, el fonema /d/ en posición final (Madrid, pared, decid...) no se realiza como oclusivo sordo, es la articulación propia de los estudiantes cuya lengua materna es el catalán.

e) La diferencia de las combinaciones silábicas posibles. Así, por ejemplo, en español las consonantes $b r$ entre vocales y en posición interior ( $a b r a z o$ ) forman parte de la misma sílaba lo que constituirá un escollo para estudiantes cuya lengua materna no admita grupos consonánticos en posición de ataque silábico, como sería el caso del árabe.

f) La existencia de procesos fonológicos propios de cada lengua. En español, por ejemplo, el sonido fricativo alveolar sordo se sonoriza cuando entra en contacto con un sonido consonántico sonoro (desde, es bueno) pero no entre vocales (es asî). Los estudiantes catalanes, por interferencia, tienden a realizar sonorizaciones incluso en ese contexto reproduciendo una característica fonológica de su lengua.

En la enseñanza de la pronunciación del español, debe perseguirse además la utilización adecuada de los elementos suprasegmentales, evitando caer en la monotonía del discurso, ya que tienen un papel relevante en la transmisión, no solo de los contenidos, sino también de la intención del hablante (Vilà et alii, 2002: 28). Por ejemplo, un enunciado como Adónde vas puede entenderse como una mera pregunta, como una exclamación de sorpresa o como un reproche, según la entonación con que sea emitido. No debe descuidarse tampoco el valor de las pausas: una pausa situada delante de un determinado elemento del discurso puede 
emplearse para realzar la información que aporta (Aguilar et alii, en prensa). En un acto comunicativo se debe prestar atención a todos los aspectos propios de la pronunciación.

\subsection{Materiales existentes. Materiales necesarios}

En las obras que tratan de la expresión oral deberían considerarse las características segmentales, que están relacionadas con la pronunciación de los sonidos, y las características suprasegmentales, recursos vocales que se superponen a más de un sonido (el acento, la entonación, la velocidad de elocución, las pausas y el ritmo); la descripción articulatoria de los elementos segmentales suele ser breve y superficial.

A modo de ejemplo, en el manual de expresión oral de Larousse (Gimeno et alii, 2000) solo aparece información referente al punto de articulación de las consonantes, y no sobre el modo de articulación. Además, no se tratan todos los sonidos; así, se ocupa de la pronunciación de la $/ \mathrm{d} /$, pero no de la $/ \mathrm{b} / \mathrm{y}$ de la $/ \mathrm{g} /$, que pertenecen a la misma clase natural. En el manual de Cuervo y Diéguez (1991) solo se distingue entre sonidos vocálicos y consonánticos, pero dentro de las consonantes sólo se explica la articulación de los sonidos nasales. De hecho, los autores ya advierten de que "la pronunciación ya se ha aprendido mediante imitación" y lo que se pretende es "mejorar la dicción y corregir algunos errores bastante frecuentes" (pág. 36). En otras obras se ofrece una descripción muy general de cómo se pronuncian algunas consonantes, dando por supuesto que el lector posee nociones básicas sobre articulatoria. Así, el Manual de estilo de TVE (Mendieta, 1993: 114) describe la pronunciación de la consonante $/ \mathrm{b} /$ de manera que el lector debería tener nociones de fonología. Se da la circunstancia además de que el rasgo del catalán que menciona es propio únicamente de algunas variedades dialectales:

B. La $b$ y la $v$ no son fonemas distintos, como ocurre, por ejemplo, en otros idiomas (catalán), donde la $v$ se pronuncia de modo semejante a la $f$, pero con vibración de la cuerdas vocales. En castellano la $b$ y la $v$ se pronuncian [b].

Sin embargo, todos los manuales coinciden en registrar una serie de errores frecuentes sobre la pronunciación de determinados sonidos de la lengua. A excepción de algunos manuales, como el de Gómez Torrego (2002), el de Alcoba et alii (2000) o el libro de estilo de Telemadrid (1993), que son especialmente completos en este aspecto, la mayoría de ellos solo tratan de aspectos muy generales como el yeísmo, el seseo o el ceceo, dedicando una o dos frases a la descripción de cada fenómeno. Por ejemplo, algunas de las afirmaciones que se recogen son:

El ceceo [...] se considera pronunciación un tanto burda. (Mendieta, 1993: 115)

El sonido de la consonante g, cuando aparece en sílaba trabada, como en ignorar, dogma, cognitivo, etc., debe pronunciarse como gue y no como je. (Gimeno et alii, 2000: 44)

También es frecuente dar sugerencias o consejos sobre una determinada pronunciación: 
Algunas consonantes en posición final $(D, Z, S)$. Nunca hay que exagerar su dicción, ya que, en tal posición, el habla permite cierto relajamiento; pero no deben suprimirse ni equipararse. (Cuervo y Diéguez, 1991: 39)

La n delante de $\mathbf{s}$ y otra consonante tiende a perderse en la pronunciación espontánea y rápida. Lo recomendable, sin embargo, sería pronunciar una $n$ aunque fuera suavizada, ya que su eliminación completa es incorrecta. (Gimeno et alii, 2000: 45)

Finalmente, algunos manuales también incluyen ejercicios, tanto para evaluar el contenido teórico explicado (Alcoba et alii, 2000; Gómez Torrego, 2002) como para mejorar la dicción (Cuervo y Diéguez, 1991; Gimeno et alii, 2000). En este último caso, se debe tener especial cuidado en no incluir ejercicios que propongan una dicción errónea, como ocurre en el manual de Larousse (Gimeno, et alii, 2000), donde se propone "la lectura cuidada en voz alta de una serie de fragmentos en los que se han seleccionado palabras que contienen el mismo sonido".

A continuación se reproduce el fragmento propuesto en este manual para la pronunciación de la $n$ como un sonido en que "la lengua entra en contacto con los alveolos".

Aunque nunca nadie haya navegado continuamente desde su nacimiento, entiende la emoción contenida en las canciones de la mar. (Gimeno et alii, 2000: 38)

Como se puede apreciar, la nasal de primera palabra que aparece en el texto anterior no se articula de modo natural como alveolar, sino velarizada por influencia de la consonante velar que le sigue.

En cuanto a los aspectos suprasegmentales de la expresión oral, no se da el mismo tratamiento a todos los fenómenos. Así, la entonación se trata, de una forma u otra, en todos los manuales, mientras que, por ejemplo, el ritmo no se trata en casi ninguno. También existe una clara diferencia en el tratamiento que se realiza en los manuales de estilo, dirigidos a locutores de radio y televisión, frente al resto de las obras aquí tratadas.

Aunque en el Libro de estilo de Telemadrid (1993), por ejemplo, se incluye una lista de palabras frecuentemente mal acentuadas, generalmente los manuales de estilo acostumbran a tratar los problemas o errores relacionados con la entonación, y en algunos casos se dan recomendaciones para realizar una buena lectura, aunque en ningún caso se facilitan instrucciones precisas sobre cómo debe hacerse. Por ejemplo:

El tono: a) debe ser coloquial; b) debe huir de la monotonía; c) no debe ser declamatorio, ni enfático, ni solemne, ni triunfalista, ni didáctico, ni afectado, ni pedante. (Mendieta, 1993: 116)

El ritmo de la locución debe adecuarse al género que se desarrolle: las noticias se deben leer con buen ritmo y un cierto estilo de urgencia [...]. (Canal Sur, 1991: 68)

En el resto de los manuales de expresión oral, se trata la entonación teniendo en cuenta su función lingüística -sobre todo, la modalidad oracional- y extralingüística -estado de ánimo del hablante, intencionalidad, etc.- (Alcoba et alii, 2000; Gimeno et alii, 2000; Cuervo y Diéguez, 1991); los casos de doble acentuación y de palabras mal pronunciadas debido al acento (Gómez Torrego, 2002; Alcoba et alii, 2000); el uso de las pausas (Alcoba et alii, 
2000; Gimeno et alii, 2000) y, únicamente en el manual de Alcoba et alii (2000) se tratan, además, otros aspectos suprasegmentales como la velocidad de elocución, la intensidad o el ritmo.

Por último, pocos son los manuales que proponen ejercicios tanto para la evaluación de los contenidos que se explican (Alcoba et alii, 2000), como para mejorar algunos de los aspectos prosódicos mencionados (Cuervo y Diéguez, 1991).

Teniendo en cuenta los materiales que se han comentado, sería necesaria la existencia de obras que ofrecieran una detallada descripción de los elementos segmentales y suprasegmentales, una sistematización de errores y una propuesta de ejercicios con soluciones para que el lector pueda autoevaluarse.

Así, la descripción de los sonidos del español debe realizarse atendiendo a sus características articulatorias y, en el caso de los elementos suprasegmentales, es imprescindible que se incluyan descripciones precisas sobre su función lingüística y sobre cómo se modula la voz para obtener la finalidad comunicativa deseada.

En cuanto a la presentación de los errores frecuentes, debe existir una sistematización que permita al lector detectar sus propios problemas -si es que los tiene- acompañada de las correcciones correspondientes, tanto a nivel segmental como suprasegmental.

Los ejercicios deben ser formulados de manera clara y sencilla, y deben ajustarse a los contenidos presentados en el manual. También es imprescindible que se incluya un solucionario para que el lector se autoevalúe y se remita, si es necesario, al apartado correspondiente.

Finalmente, un manual debería complementarse, a ser posible, con muestras sonoras de los ejemplos que se hayan citado, de los problemas mencionados y de los ejercicios que lo requieran (en soporte analógico o digital), de forma que sirvan como ilustración de los fenómenos que se manifiestan en la expresión oral.

\section{La enseñanza de la pronunciación en español como lengua extranjera}

\subsection{La enseñanza de la pronunciación en ELE: situación actual en el diseño curricular (Instituto Cervantes, Marco Europeo de Referencia) y en la evaluación (DELE)}

Al igual que sucede en otros campos como la gramática o el léxico, la enseñanza de la pronunciación en español como lengua extranjera no puede abordarse sin haber establecido previamente unos objetivos y unos contenidos, tarea propia de un diseño curricular. Así, en el que, sin duda, constituye un documento de referencia, el Plan Curricular del Instituto Cervantes (Instituto Cervantes, 1994), se definen, dentro del apartado denominado "Lengua y sistema: Contenido gramatical", los contenidos correspondientes al plano fónico para cada uno de los niveles establecidos: inicial, intermedio, avanzado y superior.

Tanto el nivel inicial como el intermedio incluyen un apartado descrito como "Pronunciación de los sonidos", mientras que en el avanzado lo encontramos como "Realización fonética de los fonemas españoles"; el nivel superior no contiene, en la definición de los contenidos, ninguna referencia a los elementos segmentales. Los contenidos del nivel inicial se centran en la identificación y producción de los sonidos vocálicos, combinaciones de vocales, sonidos consonánticos y grupos consonánticos; los del intermedio son, en cambio, la "identificación y producción de las variantes: contextuales; en función de la posición", 
definición que se repite en el nivel avanzado bajo el epígrafe "Realización fonética de los fonemas españoles".

Nótese, en primer lugar, que los contenidos del nivel intermedio y los del avanzado son idénticos, bajo títulos diferentes. La diferencia entre "pronunciación de los sonidos" y "realización fonética de los fonemas" no parece responder más que a un modo más o menos técnico de presentar los mismos fenómenos. En segundo lugar, si los niveles intermedio y avanzado contemplan las "variantes contextuales" y las "variantes en función de la posición", cabría considerar si en el nivel inicial únicamente se presentan los segmentos con valor fonológico, cosa que sería contradictoria con su definición como "sonidos".

La entonación se aborda en los cuatro niveles. En el inicial, el contenido se centra en la "segmentación del discurso en grupos fónicos" y en la "identificación y realización de los patrones entonativos básicos (enunciativo, interrogativo, exclamativo)", incluida en el epígrafe "Entonación y sintaxis". Este mismo apartado se encuentra en los restantes niveles, siempre con la indicación de que se estudian las modalidades entonativas que se presentan en cada uno de ellos. En el intermedio se estudian las interrogativas indirectas y las subordinadas sustantivas, adjetivas y adverbiales, el avanzado cubre únicamente los tres tipos de subordinadas y en el superior únicamente se hace alusión a "las estructuras sintácticas", añadiéndose los "matices expresivos de la entonación".

Este planteamiento de la entonación ligado a la sintaxis contrasta con la propia definición de contenidos gramaticales en el Plan Curricular. Por ejemplo, las interrogativas indirectas y las subordinadas se incluyen en el contenido gramatical del nivel inicial y no se mencionan, en cambio, en los contenidos fonéticos hasta el nivel intermedio. En otro orden de cosas, la estrecha relación que se establece entre entonación y oraciones subordinadas en el nivel intermedio y el avanzado no parece responder a una visión de la entonación relacionada con la estructuración del discurso oral o con la organización de la información en el enunciado, que serían tal vez más acordes con los planteamientos comunicativos que orientan el diseño curricular del Instituto.

Los niveles inicial e intermedio contemplan, además, contenidos relacionados con el acento y el ritmo. Si en el inicial se incluye la "localización del acento" y "el ritmo de la frase en la cadena hablada", el intermedio especifica como contenido la "acentuación de las palabras aisladas y distribución de los acentos en la frase". De nuevo, la acentuación de palabras aisladas no parece demasiado congruente con una metodología comunicativa; por otro lado, la división de contenidos entre el nivel inicial y el superior no es excesivamente clara, pues la distribución de los acentos en la frase (intermedio) es, precisamente, uno de los factores que condiciona el ritmo de un enunciado (inicial).

Se observa, por tanto, que la definición de los contenidos que deben abordarse en la enseñanza de la pronunciación tal como se presenta en el Plan Curricular del Instituto Cervantes no constituye, como sería esperable, un modelo de precisión ni de coherencia. Seguramente no es ajena a estas carencias la poca atención que se ha prestado a la enseñanza de la pronunciación y a la corrección fonética en el ámbito del español como lengua extranjera (Llisterri, 2003a) reflejada también, como se verá a continuación, en la evaluación $\mathrm{y}$ en los materiales publicados.

La determinación de los objetivos incide, naturalmente, de forma determinante en los criterios de evaluación (Llisterri, 2003b). Por centrarnos de nuevo en documentos oficiales de referencia, examinaremos las indicaciones que se ofrecen en la Guía para la Obtención 
de los Diplomas de Español correspondiente a cada uno de los tres niveles del DELE (Diploma de Español como Lengua Extranjera).

La Guía para la obtención del CEI (Certificado Inicial de Español) especifica que, en la prueba de lectura en voz alta que, junto con una conversación con el tribunal examinador, evalúa la expresión e interacción oral, el objetivo es "comprobar la fluidez de lectura (reconocimiento de palabras y de períodos, adecuada colocación de acentos, interpretación de números y abreviaturas, etc.) y nunca la corrección fonética, que no debe ser evaluada en este nivel." (apartado 4.5). El DBE (Diploma Básico en Español) contempla únicamente una conversación con los examinadores, en la que el candidato "Puede experimentar ciertas dificultades en la pronunciación de determinados sonidos, ajenos a su sistema fonético o propios del español. Su entonación puede no ajustarse en su totalidad a ninguno de los patrones existentes en español. Sin embargo, esos problemas no llegan a perturbar gravemente la comunicación". (apartado 2.4). Finalmente, para obtener el DSE (Diploma Superior de Español), se establece que el alumno "Tendrá un completo dominio de la pronunciación española. Aunque puede descubrirse su origen lingüistico, pronunciará de modo tal que puede ser entendido sin ninguna dificultad y se ajusta de modo sistemático a alguno de los patrones fonéticos existentes en español. Su entonación podrá mostrar ciertas interferencias de la lengua materna" (apartado 1.4).

Así, mientras que en el CEI no se valora, en principio, la pronunciación, el DBE requiere un dominio suficiente de los elementos segmentales y suprasegmentales que no altere la comunicación y, por su parte, el DSE supone un nivel en el que se acepta la interferencia de la primera lengua siempre y cuando no cree dificultades de comprensión a los hablantes nativos de español. De ello se desprende un enfoque de la evaluación del plano fónico centrado en las posibles interferencias en la comunicación, que concede un papel preponderante al receptor nativo. Se aprecia, así, un claro contraste con el Plan Curricular del Instituto Cervantes -responsable de la administración de los exámenes del DELE-, cuya definición de contenidos fonéticos no parece responder completamente a los planteamientos en los que se basa la evaluación.

\subsection{Materiales existentes: cursos, materiales generales y materiales en función de la L1 del estudiante. Materiales necesarios}

En la mayoría de los manuales publicados en España a los que se ha podido acceder (García y Sánchez, 1981; Sánchez et alii, 1982; Equipo Pragma, 1984; Martín Peris, 1984; Sánchez et alii, 1986; Miquel y Sans, 1989; Martín Peris y Sans, 1997; Cerrolaza et alii, 1998; Eusebio et alii, 1999) apenas se presta atención a la enseñanza de la pronunciación. En ellos se presentan la relación entre grafía y sonido, la función fonológica del acento, los patrones acentuales de las palabras en español y la relación entre modalidad oracional y patrón entonativo. Los métodos que van más allá y pasan de la descripción fonológica a la fonética son muy pocos (Calabuig, 1991; Miquel et alii, 1994; Alcoba et alii, 2001). Además, el número de ejercicios dedicados a estos temas es muy reducido, en muchas ocasiones se limitan a uno por lección.

Esta situación contrasta con la de los manuales publicados en el extranjero (Amann et alii, 1994; Blanco et alii, 2001) en los que se suele dedicar una parte considerable de las explicaciones gramaticales a la descripción de la pronunciación en español. Suelen basarse 
en el análisis contrastivo entre el sistema fonológico de esta lengua y la del estudiante; se proporciona una descripción de cómo se articulan los sonidos en español y se explican las combinaciones fonotácticas; los elementos suprasegmentales suelen abordarse ya desde las primeras lecciones.

Este contraste quizás se explique por la falsa creencia de que si el alumno aprende la lengua en el país, puede aprender a pronunciar por emulación, pues tiene a su profesor como modelo, mientras que si el aprendizaje se realiza en su país de origen, es posible que su profesor no sea hispanohablante nativo. Sin embargo, dado que los materiales publicados en España también se comercializan y se usan en el extranjero, este planteamiento parece erróneo.

Como complemento a los métodos, se han publicado toda una serie de obras cuyo objetivo es reforzar y ampliar algún aspecto del español. Para aprender a pronunciar, existen dos tipos de materiales: los que tienen en cuenta la lengua materna del alumno (Quilis y Fernández, 1964; Fernández González y de Santiago Guervós, 1997; Férnandez Díaz, 1999; Masip, 1999; Moreno Fernández, 2000) y los que prescinden de ella (Sánchez y Matilla, 1974; Siles Artés, 1994; Inchaurralde et alii, 2001; Nuño y Franco, 2001a; Nuño y Franco, 2001 b; Nuño y Franco, 2001 c; Romero Dueñas y González Hermoso, 2002; González Hermoso y Romero Dueñas, 2002). En general, ambos tipos de materiales se caracterizan por relegar al final el estudio de los elementos suprasegmentales (y apenas les dedican atención) y por soler adoptar un enfoque estructuralista y no comunicativo.

Tal como se analiza detalladamente en Llisterri (2003a), el problema de los ejercicios destinados a la práctica de la pronunciación es que generalmente se basan en el "escucha y repite", es decir, en la audición y en la imitación. Este procedimiento no resulta efectivo, ya que muchas veces el estudiante no es capaz de percibir la diferencia entre lo que escucha y lo que dice, ni entre lo que realmente se dice y lo que él cree oír. Así, el estudiante categoriza la secuencia fónica según los patrones de su propia lengua (sordera fonológica), lo que le impide adquirir la correcta pronunciación de la lengua motivo de estudio.

Por todo ello, los resultados de las investigaciones en fonética básica y aplicada deberían tenerse en cuenta en el diseño de los ejercicios de pronunciación de los métodos y materiales complementarios; por ejemplo, los estudios llevados a cabo en la aplicación del sistema verbo-tonal en la enseñanza de ELE, entre los que cabe destacar los de Sarmiento (1974 y 1975), Sarmiento et alii (1974), Jiménez et alii (1975), Sarmiento y de Vriendt (1977).

\subsection{El estándar oral en la enseñanza de ELE. Repercusiones en la formación de pro- fesores}

Todos los profesores de español como lengua extranjera han de plantearse qué modelo de lengua enseñan a sus alumnos, tanto en los diversos aspectos gramaticales y comunicativos como en los referentes a la pronunciación (Fernández Moreno, 1997; Saralegui, 1998). Seguir una norma permite abordar la tarea docente bajo unas directrices claras con las que conducir el desarrollo de las destrezas orales y escritas de los estudiantes. Concretando en la expresión oral, adoptar un estándar es sin duda ventajoso. Al ser una variante compartida por la comunidad lingüística, actuaría de elemento unificador que daría coherencia a la labor del conjunto del profesorado: un mismo modelo de pronunciación de los elementos segmentales y suprasegmentales y un mismo referente para corregir los errores producidos 
por los estudiantes; estos tendrían acceso a un habla común en el uso -además de socialmente prestigiosa-, que les facilitaría adquirir la competencia necesaria para comunicarse en español con independencia del centro docente en el que cursen sus estudios y del profesor que les enseñe.

No obstante, todas estas afirmaciones - no carentes de idealismo- han de ser matizadas. Como se ha apuntado antes, es impensable proponer una norma oral única para el español. Atendiendo a la situación comunicativa en la que se desarrolla el aprendizaje, los alumnos de español como lengua extranjera recibirán la norma que usen sus profesores, la que transmitan los medios de comunicación a los que tengan acceso y la que hable la comunidad lingüística en la que estén inmersos si estudian en un país hispanohablante.

En este último caso, se ha de tener en cuenta un factor que incide en el aprendizaje: los alumnos no pueden sustraerse al contacto con las variantes no normativas que existen en cada territorio; los fenómenos peculiares del habla pueden interferir en su comprensión oral $\mathrm{y}$, por supuesto, en su pronunciación, ya que en buena medida esta se formará con los datos del entorno. Se trata, como indica Blecua (2001), de "reconocer en la enseñanza [...] una verdad normal en la lingüística: la lengua como una unidad llena de tensiones, unas muy establecidas [...], otras ya dilucidadas o en trance de dilucidarse en pocos años".

Por tanto, aunque en una primera etapa quizás sea más conveniente circunscribir la enseñanza a la de una única variante, normativa, resultará imprescindible contemplar también las variaciones sociales y locales, especialmente, las características diatópicas con amplia extensión territorial (seseo-ceceo, yeísmo, aspiración, etc.), no para que los estudiantes las incorporen en sus producciones orales sino para ampliar su conocimiento y mejorar su comprensión del español. Evidentemente, conseguir que los alumnos de español como lengua extranjera alcancen una buena expresión oral requiere una formación especializada del profesor. Ya se ha mencionado que esta debe incluir conocimientos de fonética y de la descripción particular de su lengua, pero se debe puntualizar que, además de dominar un estándar, se han de conocer la complejidad de los fenómenos del español hablado, la aceptación social de las distintas variantes y los contextos en los que son adecuadas, puesto que, en definitiva, se trata de guiar a los estudiantes en el buen uso y en la correcta comprensión del español. Para cubrir estos requerimientos de la formación del profesorado sería necesario disponer de descripciones exhaustivas y actualizadas de las distintas normas, especialmente de las que se consideren estándar, y de buenas obras de consulta para la resolución de dudas.

\section{Conclusiones}

$\mathrm{El}$ análisis de los materiales revisados en este artículo muestra la falta de atención que se presta, por lo general, a las descripciones relacionadas con la expresión oral. Este hecho puede deberse a la imprecisión e incoherencia de los objetivos curriculares de la enseñanza del español, como lengua materna y como lengua extranjera. Hay una carencia de documentos normativos oficiales que recojan los usos actuales de la lengua española: variedades dialectales, estilísticas y de registro. Las obras de consulta para los nativos carecen de descripciones sistemáticas y exhaustivas; incluso en muchos casos contienen errores y ambigüedades que confunden al lector y le obligan a recurrir a obras especializadas, especialmente a estudios fonéticos. También suelen darse consejos sobre aspectos concretos de la pronunciación sin presentar ninguna técnica que permita alcanzar el pro- 
pósito que se sugiere. En el campo de la enseñanza del español como lengua extranjera faltan materiales que respondan a planteamientos comunicativos para el aprendizaje de la pronunciación y materiales metodológicamente adecuados para emplearlos en la corrección fonética.

La creación de materiales útiles para la enseñanza de los aspectos fónicos de lengua -para nativos y para extranjeros- requiere la colaboración de especialistas pertenecientes a distintas disciplinas: expertos en la descripción fonética del español, profesionales con necesidades específicas en el uso del español como lengua propia y profesores de enseñanza de EL1 y ELE.

\section{Referencias bibliográficas}

Aguilar, L., S. Alcoba, C. Carbó y M. Machuca (en prensa): "Los marcadores discursivos en la lengua oral informativa". Actas del Congreso Internacional de Análisis del discurso: lengua, cultura y valores, Pamplona, Universidad Católica de Pamplona.

Alcoba, S. (coord.), L. Aguilar, S. Luque y M. Machuca (2000): La expresión oral. Barcelona, Ariel. Alcoba, S. (coord.), C. Carbó, A. I. García, M. Inglés, A. Iruela y N. Soriano (equipo lingüístico) (2001): Es español, Madrid, Espasa.

Alvar, M. (1983): La lengua como libertad. Madrid, Cultura Hispánica.

Amann, K. A., S. Marín y E. Osorio (1994): Encuentros, Berlín, Cornelsen.

Blanco, J. A., M. A. Dellinger, P. Donley, M. I. García (2001): Vistas. Introducción a la lengua española. Boston, Vista Higher Education.

Blecua, J. M. (2001): "Unidad, variedad y enseñanza", Actas electrónicas del II Congreso Internacional de la Lengua Española, "El español en la Sociedad de la Información", http: // cvc.cervantes.es/obref/congresos/valladolid/ponencias/unidad_diversidad_del_espanol/1_la_norma_hispanica/blecua j.htm (03-11-2003).

Calabuig, M. J. (coord), F. Castro, F. Marín, R. Morales y S. Rosa (1991): Ven. Madrid, Edelsa.

Cerrolaza, M., O. Cerrolaza y B. Lloret (1998): Planet@, ele. Madrid, Edelsa.

Cuervo, M. y J. Diéguez (1991): Mejorar la expresión oral. Madrid, Narcea S. A. Ediciones.

De Luca, M. O. P. (1983): Didáctica de la lengua oral. Metodología de enseñanza y evaluación. Buenos Aires, Kapelusz.

Equipo Pragma (1984): Para empezar. Madrid, Edelsa.

Eusebio, S., Z. Fernández, C. Fernández y B. Sarralde (1999): Escala (Nivel inicial-intermedio). Madrid, Edinumen.

Férnandez Díaz, R. (1999): Prácticas de fonética para hablantes de portugués. Madrid, Arco/ Libros.

Fernández González, J. y J. de Santiago Guervós (1997): Fonética básica del español para anglohablantes. Salamanca, Plaza Universitaria Ediciones.

Fernández Moreno, F. (1997): “¿Qué español hay que enseñar? Modelos lingüísticos en la enseñanza de español/LE". Cuadernos Cervantes de la Lengua Española, 14, págs. 7-15.

García Madrazo, P. y C. Moragón Gordon (1989): Hablar y escribir. Madrid, Pirámide.

García, N. y J. Sánchez (1981): Español 2000. Madrid, SGEL.

Gimeno, I., C. Rosell y N. Pino (2000): Larousse expresión oral. Barcelona, Larousse.

Gómez Torrego, L. (2002): Manual de español correcto. Madrid, Arco/Libros.

González Hermoso, A. y C. Romero Dueñas (2002): Fonética, entonación y ortografía. + de 350 ejercicios para el aula y el laboratorio. Madrid, Edelsa. 
Hernández Alonso, C. (2001): “QQué norma enseñar?”. Actas electrónicas del II Congreso Internacional de la Lengua Española, "El español en la Sociedad de la Información", http: // cvc.cervantes.es/obref/congresos/valladolid/ponencias/unidad_diversidad_del_espanol/1_la_norma_hispanica/hernandez_c.htm (03-11-2003).

Inchaurralde, C., M. C. Saíz, A. Nocito y U. A. Kaunzner (2001): Los sonidos del español. Ejercicios de pronunciación con grabaciones. Zaragoza, Mira Ediciones SA.

Instituto Cervantes (1994): La enseñanza del español como lengua extranjera. Plan Curricular del Instituto Cervantes. Alcalá de Henares, Instituto Cervantes.

Jiménez, P., J. A. Sarmiento y E. Koberski (1975): "Utilización del sistema verbo-tonal de corrección fonética en la enseñanza del español a francohablantes", Boletín de la AEPE, 6, 10: págs. 39-45.

Kostouli, T. (2002): "Teaching Greek as L1: Curriculum and Textbooks in Greek Elementary Education", L1-Educational Studies in Language and Literature, 2, págs. 5-23.

Llisterri, J. (2003a): "La enseñanza de la pronunciación", Cervantes. Revista del Instituto Cervantes en Italia, 4, 1, págs. 91-114.

Llisterri, J. (2003b): "La evaluación de la pronunciación en la enseñanza del español como segunda lengua". En Reyzábal, M.V. (dir.): Perspectivas teóricas y metodológicas: Lengua de acogida, educación intercultural y contextos inclusivos. Madrid, Dirección General de Promoción Educativa, Consejería de Educación, Comunidad de Madrid, págs. 547-562.

Marco de referencia europeo para el aprendizaje, la enseñanza y la evaluación de lenguas. Traducción de Alejandro Valero Fernández de Common European Framework of Reference for Languages: Learning, Teaching, Assessment. Strasbourg: Council of Europe, 2001: http: //cve.cervantes.es/obref/marco/ (03-11-2003).

Martín Peris, E. (1984): Vamos a ver... Para entendernos en español. Madrid, Edelsa/Edi6. Martín Peris, E. y N. Sans (1997): Gente. Barcelona, Difusión.

Martínez de Sousa, J. (1996): Diccionario de usos y dudas del español actual. Barcelona, Biblograf. Masip, V. (1999): Gente que pronuncia bien 1. Curso de pronunciación española para brasileños.

Barcelona, Difusión.

Mendieta, S. (1993): Manual de estilo de TVE. Barcelona, Editorial Labor.

Ministerio de Educación, Cultura y Deportes, Universidad de Salamanca e Instituto Cervantes (2001): Guía para la obtención de los Diplomas de Español (DELE). Certificado Inicial de Español: http: //diplomas.cervantes.es/documentos/2/1/Certificado\%20Inicial\%20de $\% 20$ Espanol.pdf

Ministerio de Educación, Cultura y Deportes, Universidad de Salamanca e Instituto Cervantes (2001): Guía para la obtención de los Diplomas de Español (DELE). Diploma Básico de Español: http: //diplomas.cervantes.es/documentos/2/1/Diploma\%20Basico $\% 20 \mathrm{de} \% 20$ Espanol.pdf

Ministerio de Educación, Cultura y Deportes, Universidad de Salamanca e Instituto Cervantes (2001): Guía para la obtención de los Diplomas de Español (DELE). Diploma Superior de Español: http: //diplomas.cervantes.es/documentos/2/1/Diploma\%20Superior\%20de\%20Espanol.pdf

Miquel, L. y N. Sans (1989): Intercambio. Barcelona, Difusión.

Miquel, L., N. Sans y D. Poch (asesoría fonética) (1994): Rápido. Barcelona, Difusión.

Moreno Fernández, F. (2000): Ejercicios de fonética para hablantes de inglés. Madrid, Arco/Libros.

Navarro Tomás, T. (1918): Manual de pronunciación española Consejo Superior de Investigaciones Cientificas. Madrid, Instituto Miguel de Cervantes (Publicaciones de la Revista de Filología Española, III).

Navarro Tomás, T. (1928): Compendio de Ortología española para la enseñanza de la pronunciación normal en relación con las diferencias dialectales. Madrid, Hernando.

Nuño, P. y J. R. Franco (2001a): Ejercicios de fonética. Nivel Inicial. Madrid, Anaya.

Nuño, P. y J. R. Franco (2001b): Ejercicios de fonética. Nivel Intermedio. Madrid, Anaya.

Nuño, P. y J. R. Franco (2001c): Ejercicios de fonética. Nivel Avanzado. Madrid, Anaya.

Poch, D. (1999): Fonética para aprender español: Pronunciación. Madrid, Edinumen. 
Quilis, A. y J. A. Fernández (1964): Curso de fonética y fonología españolas para estudiantes angloamericanos. Madrid, CSIC.

Radio y Televisión Andaluza (1991): Libro de estilo / Canal Sur Televisión. Sevilla, Canal Sur Televisión.

Real Academia Española (1931): Gramática de la lengua española. Madrid, Espasa Calpe.

Real Academia Española (1973): Esbozo de una nueva gramática de a lengua española. Madrid, Espasa Calpe.

Real Academia Española (1992): Diccionario de la lengua española Madrid, Espasa Calpe.

Real Academia Española (1999): Ortografía de la lengua española, Madrid, Espasa Calpe.

Real Academia Española (2001): Diccionario de la lengua española, Madrid, Espasa Calpe.

Real Academia Española: Diccionario panhispánico de dudas, http: //www.rae.es

Romero Dueñas, C. y A. González Hermoso (2002): Tiempo para pronunciar. + de 100 ejercicios. Madrid, Edelsa.

Sánchez, A. (1983): Una metodología de la lengua oral. Madrid, Escuela Española.

Sánchez, A. y J. A. Matilla (1974): Manual práctico de corrección fonética del español. Madrid, SGEL.

Sánchez, A., J. M. Fernández y C. Díaz (1986): Antena 1. Madrid, SGEL.

Sánchez, A., M. Ríos y J. A. Matille (1982): Entre nosotros. Madrid, SGEL.

Saralegui, C. (1998): "A vueltas con la pluralidad de normas del español y el modelo lingüístico para la enseñanza de la pronunciación", RILCE, 14, 2, págs. 367-386.

Sarmiento, J. A. (1974): "Système de fautes et correction phonétique par la méthode verbo-tonale des francophones belges qui apprennent l'espagnol", Revue de Phonétique Appliquée, 29, págs. $51-77$.

Sarmiento, J. A. (1975): "Importancia del condicionamiento acústico en la enseñanza fonética del español como lengua extranjera", Boletín de la AEPE, 12, págs. 67-73.

Sarmiento, R., S. De Vriendt (1977): "Corrección fonética de los neerlandófonos que estudian español”, Boletín de la $A E P E, 10,17$, págs. 53-94.

Sarmiento, J. A., P. Jiménez, E. Koberski (1974): "L'application de l'appareil SUVAGLINGUA de correction phonétique à l'enseignement de l'espagnol aux francophones", Revue des Langues Vivantes, 40,7, págs. 671-695.

Seco, M. (1998): Diccionario de dudas y dificultades de la lengua española. Madrid, Espasa Calpe.

Siles Artés, J. (1994): Ejercicios prácticos de pronunciación del español. Madrid, SGEL.

Telemadrid (1993): Libro de estilo de Telemadrid. Madrid, Ediciones Telemadrid.

Trigo Cutiño, J. M. (1998): "Desarrollo de la oralidad en la educación secundaria", Revista Interuniversitaria de Formación del Profesorado, 31, págs. 35-53.

Vilà, M. (coord.), C. Ballesteros, J. M. Castellà, A. Cros, M. Grau y J. Palou (2002): Didàctica de la llengua oral formal. Continguts d'aprenentatge i seqüències didàctiques. Barcelona, Graó. 